Endocrinol. Japon. Vol. 6, No. 1 (1959)

\title{
THE EFFECT OF HYPOTHALAMIC LESIONS ON LITTER GROWTH IN RATS
}

\author{
AKIRA YOKOYAMA AND KATSUAKI ÔTA
}

Faculty of Agriculture, Nagoya University

Participation of the hypothalamus and the posterior lobe of the pituitary in the milk ejection reflex is well known. On the other hand, the importance of suckling stimulus to maintain the secretion of milk, reported by Selye (1934), suggests that the hypothalamus plays some rôle in controlling the secretion of anterior pituitary galactopoietic hormones.

An attempt was made to clarify the relationship between activities of the anterior and of the posterior lobe of the pituitary gland, which seemed to be associated with suckling stimuli through the hypothalamus, by means of hypothalamic lesions in rats. Cross and Harris (1952) reported that lesions in the S.O.H. tract in the median eminence caused a marked retardation of milk removal without disturbing the secretory activity of the mammary gland in rabbits. Recently, similar results were reported by Donovan and van Werfften Bosch (1957).

It is interesting to study whether or not hypothalamic lesions are related to milk-ejection evoked by the secretion of posterior pituitary hormone and to the secretory activity of the mammary gland controlled by anterior pituitary hormone(s). To identify which specific hypothalamic nuclei are related to the phenomena described above is one of the purposes of the present experiments.

\section{MATERIALS AND METHODS}

Wistar strain rats in the first lactation, weighing $130-200 \mathrm{~g}$, were used. They were maintained with wheat, a small amount of green food and water ad lib. Litters were limited to 6 on the 1st day of lactation, equal numbers of each sex being kept whenever possible. Hypothalamic lesions were placed bilaterally under sodium amobarbital anaesthesia with the apparatus described by Greer et al. (1955), using a unipolar electrode and a direct current of 2-3 mA. for 30 secs. In a sham operation, the electrode was inserted without any current. The operation was carried out on the 7 th day of lactation. Careful observation on nursing behaviour and weighing of total litters and mothers were continued every morning until the day of sacrifice. Palpation of the mammary gland was also done to evaluate the rate of milk secreting activity and involution. Animals were usually autopsied on the 20th day of lactation. Animals which failed to rear their young, however, were usually autopsied as soon as the failure was well confirmed. In some cases, new off spring of about the same age were replaced as a foster litter after the death of the mother's own litter.

The brain was fixed by perfusing with 10\% formalin. Anterior and posterior lobes of the pituitary glands were weighed after $24 \mathrm{hrs}$. of fixation in a $10 \%$ formalin solution. However, in several cases, they were weighed and the anterior lobes were prepared for prolactin assay.

Received for publication December 22, 1958. 
Serial sections $(8 \mu)$ of hypothalami were made and stained with Kurotsu-Katayama's carbolthionine method and examined histologically to determine the site of lesions. Ovaries were weighed, fixed in a 10\% formalin solution for histological study. The left abdominal mammary gland was weighed after $24 \mathrm{hrs}$. of fixation in Bouin's fluid for histological observations. Details of histological studies of the ovaries and mammary glands and the results of the prolactin assay will be reported elsewhere.

\section{RESULTS AND DISGUSSION}

After the operation, all the animals recovered well from anaesthesia and seemed to be in good condition during the experimental period. In the sham operated group, five of the animals nursed their young satisfactorily and exhibited almost normal milk yield which was estimated by the increase in litter weight, this being observed even on the day following the operation. There was no significant difference in the regression coefficient of the growth curves between the normal groups and the sham operated group (Fig. 1).

Animals bearing hypothalamic lesions were divided into the following two groups on the basis of the location of the lesions: 1. animals having anterior hypothalamic lesions (Group A). 2. animals having lesions in the medial part of hypothalami (Group M). Each group was classified into 2 subgroups according to their ability to rear their young.

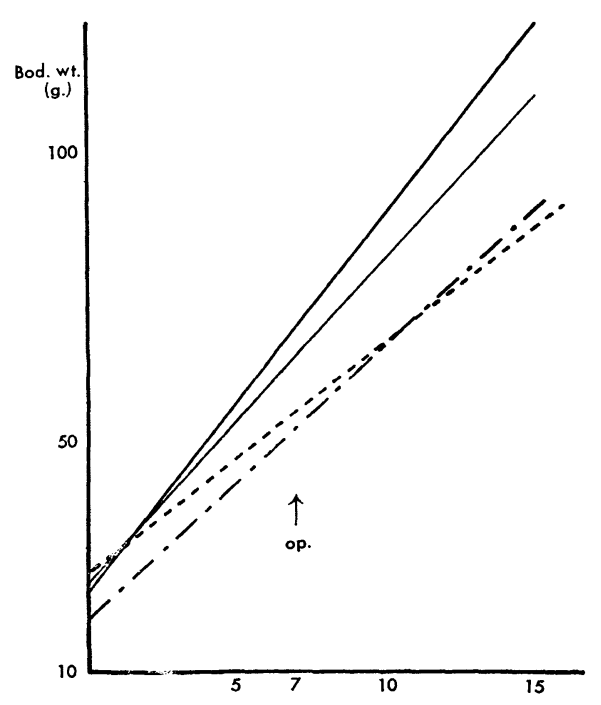

Fig. 1 Growth curves of the litters of each group. Day of the operation is illustrated by op.

Normal Group ( $Y=6.4753 X+23.738)$, Sham operated Group $(Y=5.4524 X$ $+25.550)$, - - Group $A-1(Y=4.6259 X$ $+19.4594), . . . . .$. Group $M-1 \quad(Y=3.9363 \mathrm{X}$ $+27.2787)$.
Seven out of 17 animals, with anterior hypothalamic lesions, succeeded in rearing their young (Group A, 1). This group took care of their offspring and nursed satisfactorily after the operation. Although the offspring of these animals suckled vigorously and were able to increase their body weight, the rate of increase was significantly lower than that of the normal group $(\mathrm{P}<0.001)$ (Fig. 1). The lesions in this group were found in the area just dorsal to the optic chiasma and/or in the area lateral to the supraoptic area. In some cases, the Nucl. suprachiasmaticus and the Nucl. supraopticus were destroyed unilaterally, however, the Nucl. paraventricularis and the periventricular area were usually completely spared.

In another group (Group A-2), the animals became savage after the operation, failed to nurse and sometimes even ate their young. They 


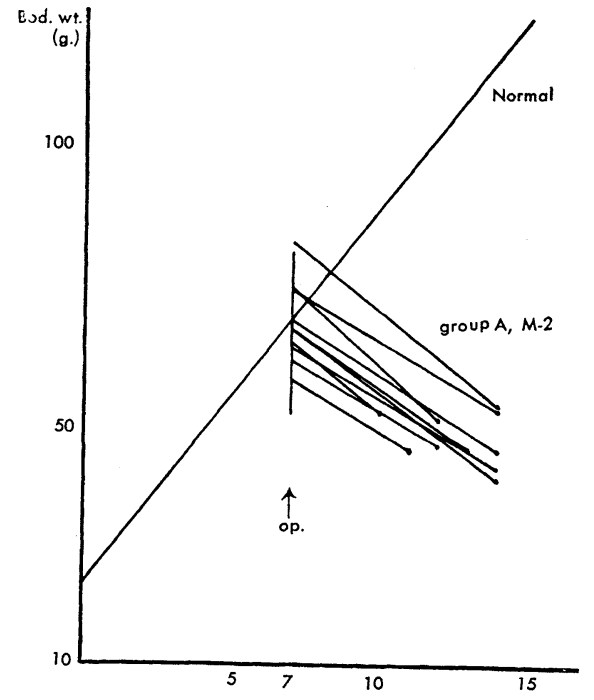

Fig. 2 Growth curves of the litters of Group A-2, M-2. Day of the operation is illustrated by op. Parpendicular line crossing the growth curve of normal litters shows the fiducial limit $(95 \%)$ on 7 th day of lactation. Each declining line corresponds to the decrease in body weight of the individual litter.

was easily squeezed out of their teats by soft palpation 2 or 3 days after the operation.

In comparing the site of hypothalamic lesions of this subgroup with that of the foregoing subgroup, it seemed to be necessary to destroy the periventricular area of the medial preoptic area or a part of the Nucl. paraventricularis in order to impair the ejection reflex. The significance of the latter in oxytocin secretion and in milk ejection was reported by Olivecrona (1954), Andersson (1951 a, b) and Cross (1955). However, the significance of the Nucl. supraopticus or the Nucl. supraopticus in the milk ejection reflex could not be clearly elucidated from these results.

Eight out of 25 operated mothers, with lesions in the medial part of hypothalami, reared their young successfully (Group M-1). The increase in body weight of these litters was significantly lower than normal litters $(\mathrm{P}<0.001)$ and Group A-1 $(\mathrm{P}=0.02)$ (Fig. 1). It must be taken into consideration that changes in the constituents of the milk, as reported by Donovan and Van Der Werff Ten Bosch (1957), may be responsible for the low growth rate of the rats in Group A-1, and M-l rather than a decrease in the milk yield. However, specific study concerning this point was not performed.

The lesions in this group were smaller than those observed in the other groups, and a part of the Nucl. dorsomedialis and the Nucl. ventromedialis were destroyed 


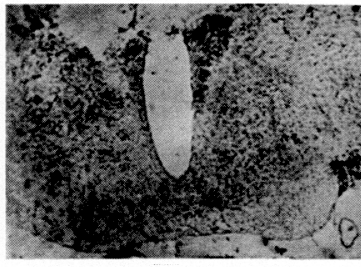

a

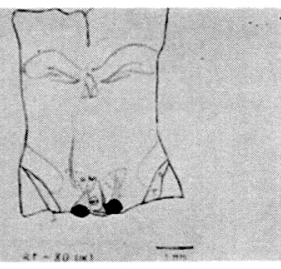

b
Fig. 3. A projection and a photomicrograph of a section through the hypothalamus in a rat. a. Lesions of a rat that failed to rear her young. The unilateral paraventricular nuclei and its dorso-caudal part were destroyed. Supra optic nuclei appeared in the right lower side of this photograph. b. Lesions of a rat that failed to rear her young. The bilateral arcuate nuclei and the unilateral ventromedial unclei of the hypothalamus were destroyed.

Note: The Number that follows Rt indicates the rat number, while the number in parenthesis shows the number of the serial sections. A: Arcuate nuclei, AC: Anterior commissure, DM: Dorsomedial nuclei of the hypothalamus, FX: Fornix, OCH: Optic chiasm, OT: Optic tract, VM: Ventromedial nuclei of the hypothalamus
(Fig. 4 b). Therefore, even if these lesions destroyed the specific zone related to the milk ejection reflex (especially oxytocin secretion), it seems unlikely that they were large enough to impair the reflex arc completely. Partial destruction of the Nucl. arcuatus was found not only in the animals of this group, but also in the animals which failed to nurse their young (Group M-2) (Fig. 3b, 4c). The median eminence was completely spared.

The remaining 17 rats, with lesions in the medial part of the hypothalami, failed to rear their young (Group

M-2), though the nursing behaviour of these animals was normal. The mammary glands of this group were filled with milk for more than 2 or 3 days after the operation and then seemed to commence the regressional change gradually; however, milk could be squeezed from their teats. These findings were confirmed by histological studies. Relatively compact alveoli systems filled with many fat globules and eosinophilic colloidal substances were found in the mammary glands of rats autopsied even 10 days after the operation. In addition to these findings, the author found that $1-12 \mathrm{~g}$ of milk/day could be obtained for 7-12 days by the administration of oxytocin to the rats bearing medial hypothalamic lesions (Yokoyama and Ôta, 1958). These findings were consistent with those reported by Cross and Harris (1952), who were able to obtain appreciable amount of milk by oxytocin administration after destruction of the S.O.H. tract in rabbits. It may be deduced from these observations that these lesions caused an interference in the milk ejection by destroying the hypothalamic zone specifically related to the oxytocin secretion rather than an interruption of the secretion of galactopoietic hormones from the anterior pituitary gland. However, Benson and Folley (1957) reported that oxytocin administration retarded the mammary gland involution which followed the cessation of suckling, by eliciting the release of prolactin, and this conception was supported by experiments performed by Desclin (1956). Therefore, the possibility can not completely be excluded that small amount of oxytocin, released by suckling stimuli because of an incomplete blockade of the hypothalamic pathway, was able to reach the anterior lobe; this amount of oxytocin being not enough to elicit the ejection response, but enough to cause the release of prolactin from the anterior pituitary by direct action.

The lesions of this group were located mainly in the periventricular area of 


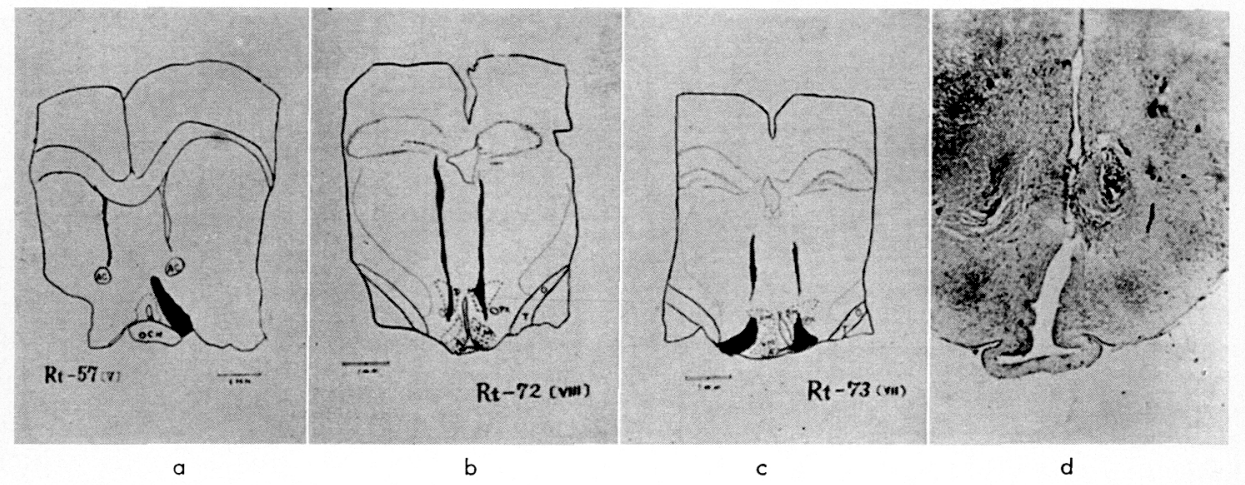

Fig. 4. Projections and a photograph of sections through the hypothalami of the rats bearing lesions. All solid black areas represent the sites of the lesions. a. Projection (X20) of a section through the anterior part of the optic chiasma of a rat that succeeded in nursing. Contralateral lesions were losated caudolaterally. b. Projection of a section of a rat succeeded in nursing, bearing medial hypothalamic lesions. The lesions did not seem to be large enough to impair the ejection reflex completely. c. Projection of the section of a rat succeeded in nursing. The unilateral arcuate nuclei were destroyed, but the contralateral nucleus was spared. Comparison of the lesions, of this animal with the one shown in Figure $3 b$, seemed to indicate that the bilateral arcuate destruction and/or the destruction of the adjacent area of this nuclei might be necessary in order to block the milk ejection reflex. d. Photomicrograph showing the lesions in the medial part of the hypothalamus in a rat that failed to rear her young. The dorsomedial nuclei and the dorsal parts of the ventromedial nuclei of the hypothalamus were destroyed. Arcuate nuclei remained intact.

the medial hypothalamic area, namely in the Nucl. ventromedialis and the Nucl. dorsomedialis (Fig. $4 \mathrm{~d}$ ). These results seemed to indicate that these 2 nuclei participated in the milk ejection reflex, and that this impression was in harmony with the theory derived from the experiments reported by Shimizu et al. (1956), Yokoyama (1956), and Fujii (1957) on the electrical stimulation of the hypothalamus in rabbits. Another confirmaiton of this present research also came from the independent studies of Fujii (1957) and Ban et al. (1957), who found that electrolytic lesions placed in these areas also caused the cessation of the ejection reflex in rats and rabbits respectively.

As described above, the unilateral Nucl. arcuatus was destroyed in 3 rats of the Group M-2. Comparison of the contralateral lesions of this group with those of the foregoing group, seemed to indicate that the bilateral destruction of the Nucl. arcuatus or the destruction of the adjacent area of this nuclei was necessary to block the milk ejection reflex. In contrast with the findings of Cross and Harris (1952) and Donovan and Van Der Werff Ten Bosch (1957), the median eminence was almost spared in this group.

Vaginal smears of Group M-2 showed typical lactational smears whenever vigorous suckling continued unabated. When the young grew weak or died, and the suckling stimulus became slight or ceased, an oestrous smear reappeared. After the oestrous smear reappeared almost all the animals in this group showed a relatively normal cycle. It may be considered that the lactational vaginal 
smear resulted from the prolactin secretion caused by the suckling stimulus as reported by Selye and McKeown (1934). In another experiment, the authors mated these animals and they delivered. During the 2nd lactation, they were unable to rear their young unless oxytocin was administered (Yokoyama and Ôta, 1958). These findings also implied the possibility of selective impairment of the part of the hypothalamus which was related to oxytocin secretion, without disturbing the production of milk, though the possibility discussed above regarding the oxytocin-prolactin relationship still remains.

Further studies will be necessary in order to draw a final conclusion.

\section{SUMMARY}

In order to investigate the hypothalamic control of lactation, bilateral electrolytic lesions were given stereotaxically in the hypothalamus of lactating rats.

Lactational performance of these rats bearing hypothalamic lesions were estimated mainly by litter growth curves.

The animals recovered quickly from the operation and were in good condition and they were divided into the following 2 groups on the basis of the location of the lesions: 1. animals with anterior hypothalamic lesions (Group A), 2. animals with lesions of the medial part of the hypothalami (Group M). Each group was classified into 2 subgroups according to their ability to rear their young. The regression coefficient of the growth curve of the litters of the animals which reared their young in both Groups $A$ and $M$ was significantly lower than those of the normal and sham operated groups.

The litters, of the animals which failed to rear their young, lost their weight gradually and died within 7 days after the operation and no milk was found in their stomachs in spite of their vigorous suckling; however, milk could be squeezed from the mother's teats even 10 days after the operation.

A lactational vaginal smear was obtained whenever suckling lasted, and after cessation of the suckling stimulus an oestrous smear reappeared within a few days.

These findings imply the possibility of selective impairment of the part of the hypothalamus, which is linked to the secretion of oxytocin.

\section{ACKNOWLEDGEMENTS}

The authors wish to express their sincere thanks to Prof. S. Nakajo for his encouragement and to Prof. M. Yasuda, Nagoya University, and Prof. M. Naito, Tokyo University, for their helpful advice and constructive criticism throughout this study. Our thanks are also extended to Mr. T. Nishida for his skilled technical help and thoughtful advice.

\section{ADDENDUM}

After this manuscript had been sent to the Endocrinologia Japonica, we found that similar experiments had been carried out by Drs. S.M.McCann, R.Mack 
and C.Gale. They reported that lesions in the supraoptico-hypophysial tract of hypothalamus blocked both milk ejection and milk production in rats.

\section{REFERENCES}

Andersson, B. (1951, a). Acta physiol. Scand. 23, 1.

Andersson, B. (1951, b). Acta physiol. Scand. 23, 8.

Ban, T., S. Shimizu and H. Tanimura (1957). Clinical. Endocrinology 5, 948. (In Japanese)

Benson, G. K. and S. J. Folley (1957). J. Endocrinol. 16, 189.

Cross, B. A. (1955). J. Endocrinol. 12, 15.

Cross, B. A. and G. W. Harris (1952). J. Endocrinol. 8, 148.

Desclin, L. (1956). Compt. rend. soc. biol. Paris, 150, 1489.

Donovan, B. T. and J. J. Van Der Werff Ten Bosch (1957). J. Physiol. 137, 410.

Fujii, K. (1957). 9th Annual Meeting of Japanese Obstetrics and Gynecological Society, pp. 76-95. (In Japanese)

Greer, M. A., C. Mencken, and K. T. Bolen, (1955). Proc. Soc. Exptl. Biol. Med. 89, 480.

McCann, S. M., R. Mack and C. Gale (1958). Federation Proc. 17, 107.

Olivecrona, H. (1954). Nature 173, 1001.

Selye, H. (1934) Am. J. Physiol. 107, 535.

Selye, H. and T. McKeown (1934). Surg. Gynecol. and Obstet. 59, 886.

Shimizu, S., T. Ban and T. Kurotsu (1956). Med. J. Osaka Univ. 7, 79.

Yokoyama, A. (1956). Japanese J. Animal Reproduction 2, 44. (In Japanese)

Yokoyama, A. and K. Ota (1958). Japanese J. Zootechnical Sci. 29, Suppl. 7. (In Japanese) 